\title{
ANÁLISIS DEL EFECTO DE SECUENCIAS DE CULTIVO SOBRE EL ESCURRIMIENTO SUPERFICIAL Y PÉRDIDAS DE SUELO Y HERBICIDAS
}

\author{
Seehaus, m.S. ${ }^{1 ;}$ Sasal, M.C. ${ }^{1} ;$ Van Opstal, N. ${ }^{1}$; Gabioud, e.A. ${ }^{1}$; \\ WILSON, M.G. ${ }^{1 ;}$ WINGEYeR, A. ${ }^{1} ;$ MICHLIG, M.P. ${ }^{2,3} \&$ RePETTI, M.R. ${ }^{3}$
}

\begin{abstract}
RESUMEN
La evaluación del efecto de los sistemas agrícolas sobre el ambiente es fundamental para minimizar sus impactos negativos. Este estudio evalúa las pérdidas de agua, sedimentos y herbicidas por escurrimiento en distintas secuencias agrícolas, luego de una precipitación próxima a la pulverización. Fue realizado en parcelas de escurrimiento bajo lluvia natural, en INTA Paraná. Las secuencias de cultivo evaluadas fueron: soja continua (S), soja continua con trigo como cultivo de cobertura (CC-S) y trigo/soja (T/S) bajo siembra directa, las cuales fueron pulverizadas con glifosato y cletodim. Considerando el total de los datos analizados, los porcentajes perdidos en escurrimiento de glifosato y cletodim respecto a lo aplicado oscilaron entre 5,3-13,5\% y 2,6-5,9\%, respectivamente. Las pérdidas de suelo y AMPA (concentración) fueron mayores para S. Se resalta la importancia de las condiciones meteorológicas posteriores a las pulverizaciones para reducir el riesgo de aportes difusos de agroquímicos hacia cuerpos de agua.
\end{abstract}

Palabras clave: secuencia de cultivos, escurrimiento, glifosato, AMPA y cletodim.

\begin{abstract}
Effect of crop sequence on water runoff and soil and herbicides losses.

Assessing agricultural systems effects on the environment is essential to minimize any negative impact. This study evaluates water, sediments and herbicides losses by runoff on different crop sequences due to a precipitation event following spraying of herbicides. Measurements were conducted in natural-rainfall runoff plots at INTA Paraná. Crop sequences were: soybean monoculture
\end{abstract}

1.- Estación Experimental Paraná de I.N.T.A. Ruta provincial 11 km 12,5 Oro Verde, Argentina. +54343497 5200. Email: seehaus.mariela@inta.gob.ar

2.- Consejo Nacional de Investigaciones Científicas Técnicas (CONICET), C1033AAJ Buenos Aires, Argentina. $+54114899-5400$.

3.- PRINARC, FIQ-UNL, Santiago del Estero 2654, C.P. 3000, Santa Fe, Argentina. +54 3424571161.

Manuscrito recibido el 15 de mayo de 2020 y aceptado para su publicación el 27 de julio de 2020.

Seehaus, M.S.; Sasal, M.C.; Van Opstal, N.; Gabioud, E.A.; Wilson, M.G.; Wingeyer, A.; Michlig, M.P.; Repetti, M.R. Análisis del efecto de secuencias de cultivo sobre el escurrimiento superficial y pérdidas de suelo y herbicidas. FAVE - Ciencias Agrarias 19 (2): 77-90. CC BY-NC-SA 4.0 
M.S. Seehaus et al.

(S), soybean monoculture with wheat as cover crop (CC-S), and both wheat / soybean as cash crops (T/S). All sequences were sprayed with glyphosate and clethodim before soybean's sowing. Compared to the amount applied, the proportion of glyphosate and clethodim in runoff ranged between $5.3-13.5 \%$ and $2.6-5.9 \%$, respectively. Losses of soil and AMPA (concentration) were greatest for $\mathrm{S}$. The results highlight the importance of checking meteorological forecast conditions before spraying of herbicides to reduce nonpoint pollution of water bodies.

Key words: agricultural systems, runoff, herbicides, precipitations.

\section{INTRODUCCIÓN}

La producción agrícola en la provincia de Entre Ríos sigue la tendencia a nivel nacional de las últimas décadas, con incrementos de alrededor del $100 \%$ en la superficie sembrada y $122 \%$ en la producción de soja comparando las campañas 2000/01 y $2016 / 17^{1}$. Este escenario se caracteriza por un aumento en los volúmenes utilizados de agroquímicos, especialmente herbicidas (Sasal et al., 2017). Un informe de Andrade et al. (2017) para Argentina muestra los aumentos desde la campaña 1990/91 a la campaña 2011/12 tanto en volumen de plaguicidas total utilizados como su uso por hectárea, con aumentos que rondan el 900 y $100 \%$, respectivamente. Dentro de este mercado, se destaca el glifosato como el principal plaguicida comercializado, abarcando el $62 \%$ de la cantidad comercializada de agroquímicos para el año 2014 (CASAFE 2014). Además, el uso reiterado de algunos herbicidas asociados a cultivos transgénicos propició la aparición de malezas resistentes (Taberner Palou et al., 2007). Frente a ello, muchas de las estrategias agronómicas para gestionar el problema de la resistencia de las malezas, consisten en incrementar los volúmenes de uso y en aplicar nuevas mezclas para mejorar o prolongar el control.

Montoya et al. (2017) advierten la evolución de malezas resistentes a glifosato, con una importante participación de las gramíneas, para las cuales se utilizan en forma frecuente graminicidas como cletodim y haloxifop R-metil. En el informe de la Cámara de Sanidad Agropecuaria y Fertilizantes se advierte que, a pesar de seguir siendo el glifosato el activo de mayor comercialización en Argentina, pierde participación en el mercado dentro de los herbicidas (CASAFE 2012). También, destaca la evolución del cletodim, con un crecimiento del $124 \%$ en la facturación y un $111 \%$ en el volumen comercializado para el período 2011-2012.

Diversos autores coinciden en que los plaguicidas representan potenciales fuentes de contaminación ambiental (Sasal et al., 2017). Su presencia se ha podido verificar en el aire, en el suelo, en el agua superficial y subterránea y en los alimentos (Alonso et al., 2018; Astoviza et al., 2015; Caprile et al., 2019; Lorenzatti et al., 2008; Primost et al., 2017; Seehaus, 2019; Villaamil Lepori et al., 2013) y son considerados fuente de contaminación no puntual del ambiente (Andrade et al., 2017). Si bien su uso tiene

1.- Datos extraidos de Bolsa de Cereales de Entre Ríos (http://www.bolsacer.org.ar/Fuentes/estadisticas.php). 
un determinado objetivo de control (maleza, insecto, hongo), en el proceso de aplicación una proporción variable se dispersa en el ambiente y alcanza diversos destinos "no blanco", atravesando distintos procesos de conversión y transporte (Seehaus, 2019). En lo que respecta a su impacto sobre la calidad de agua, este se origina fundamentalmente por el aporte difuso por deriva, escurrimiento o erosión desde lotes productivos y/o por aporte puntual debido a prácticas mal implementadas, como lavado de equipos aplicadores sobre cursos de agua (Sasal et al., 2017).

En Entre Ríos, las características naturales de su topografía ondulada, así como la baja capacidad de infiltración de sus suelos y las precipitaciones intensas en primavera-verano-otoño, predisponen a gran parte de la superficie provincial a procesos de degradación de los suelos, especialmente por erosión hídrica (Scotta y Paparotti, 1990), a la vez que se incrementa el riesgo de contaminación de los cursos de agua por escurrimiento desde agroecosistemas. La llegada de agroquímicos a cursos de agua a través del escurrimiento superficial, puede darse a través de su unión a sedimentos erosionados (para aquellos con alta sorción) o por disolución en agua (los de alta solubilidad). En este proceso, las lluvias son el principal factor que controla la dinámica del escurrimiento, erosión y pérdida de plaguicidas (Caprile et al., 2019). Sin embargo, hay otros factores a tener en cuenta tales como tipo y cantidad de residuos vegetales en superficie, rotación agrícola, sistema de labranza y su impacto sobre la estructura superficial de suelo, entre otros (Caprile et al., 2019; Villarreal et al., 2018).
Respecto al herbicida glifosato ( $\mathrm{N}-$ fosfonometil glicina) y su principal metabolito, el ácido amino metilfosfónico (AMPA), existe una vasta bibliografía que aporta al entendimiento de su dinámica ambiental. Es un herbicida organofosforado no selectivo, de amplio espectro y de acción sistémica postemergente que se aplica sobre el follaje, con un modo de acción que se basa en la inhibición de la síntesis de aminoácidos esenciales. Si bien es fuertemente retenido por el suelo (alto valor del coeficiente de partición carbono orgánico-agua del suelo o sedimento- Koc $6920^{2}$ ), también se lo considera muy soluble en agua (10000 $\mathrm{mg} \mathrm{l}^{-1}$ a $25^{\circ} \mathrm{C}$ el compuesto puro) (Battaglin et al., 2014). En el suelo es adsorbido mediante uniones fosfato y degradado por microorganismos, cuyo principal metabolito es el AMPA. A pesar de su relativa escasa movilidad, abundan estudios que muestran su llegada a cursos de agua superficial y subterránea (Aparicio et al., 2013; Ayarragaray et al., 2015; Etchegoyen, 2014; Lupi et al., 2019; Primost, 2013; Primost et al., 2017; Sasal et al., 2012; Sasal et al., 2017).

En cambio, la literatura científica acerca del herbicida cletodim en cuanto a su comportamiento ambiental y la persistencia en el medio ambiente es bastante limitada (Montoya et al., 2017). Es un herbicida selectivo, sistémico, que actúa a través de la inhibición de la acetilCoA carboxilasa (ACCasa). Su solubilidad es considerada alta (5450 mg 1-1 en agua a $20 \mathrm{oC}$ ) y es clasificado como móvil a muy móvil en el suelo con valores de coeficiente de partición Kfoc de $3-43^{3}$. Se lo considera no persistente en el suelo, con un DT50 que varía entre 0 a 3 días.

2.- https://sitem.herts.ac.uk/aeru/footprint/es/Reports/2395.htm

3.- https://sitem.herts.ac.uk/aeru/footprint/es/Reports/164.htm\#none 
Antecedentes previos muestran resultados distintos respecto a la influencia de sistemas de labranza, rotaciones y tipo de residuos o rastrojo sobre el suelo, sobre las pérdidas de plaguicidas (Warnemuende, 2007). Sin embargo, hay coincidencia respecto a la importancia de los eventos de precipitación cercanos a la fecha de aplicación y su influencia sobre su escurrimiento fuera del lote (González, 2018; Oszust et al., 2015; Sasal et al., 2015; Screpanti et al., 2005; Shipitalo et al., 1997). De esta manera, se encuentran estudios de monitoreo donde se registra un mayor número de detecciones de plaguicidas en cursos de agua en momentos con mayores caudales y eventos extremos de precipitaciones (Vázquez-Amabile et al., 2017), y otros que atribuyen menores concentraciones asociadas a efecto dilución en los cursos de agua (Sasal et al., 2017).

En este marco, el estudio de los efectos sobre el ambiente de las prácticas agrícolas y la comprensión de los mecanismos a través de los cuales los plaguicidas alcanzan distintos compartimentos ambientales, constituye el paso inicial para las propuestas de minimización de los impactos asociados. El objetivo del presente trabajo fue evaluar el efecto de distintas secuencias de cultivo sobre las pérdidas de agua, sedimentos y glifosato y cletodim por escurrimiento, luego de un evento de precipitación inmediatamente posterior a la aplicación de dichos herbicidas.

\section{MATERIALES Y MÉTODOS}

\section{Sitio de estudio}

El estudio fue realizado en la Estación Experimental de Paraná del Instituto Nacional de Tecnología Agropecuaria (INTA) de la provincia de Entre Ríos ( $31^{\circ} 51^{\prime} \mathrm{S}$ y $60^{\circ} 31^{\prime} \mathrm{W}$ ), en parcelas de escurrimiento bajo lluvia natural con diferentes secuencias de cultivo bajo siembra directa. La región tiene clima subhúmedo (lluvia anual $\approx 1000 \mathrm{~mm}$ ) y templado (temperatura anual $\approx 18,3^{\circ} \mathrm{C}$ ). El $75 \%$ de las lluvias anuales ocurren en primavera y verano (Sasal et al., 2009). Las temperaturas del invierno raramente son inferiores a $0^{\circ} \mathrm{C}$.

Las parcelas se encuentran sobre un Argiudol ácuico fino, illitico, térmico (US Soil Taxonomy, 1999) de la Serie Tezanos Pinto (Luvic Phaeozem, WRB). La textura del horizonte A $(17 \mathrm{~cm})$ es franco limosa con 270 y $660 \mathrm{~g} \mathrm{~kg}^{-1}$ de arcilla y limo, respectivamente (Plan Mapa de Suelos, 1998). Presentan dimensiones de $4 \mathrm{~m}$ de frente $\mathrm{y}$ $25 \mathrm{~m}$ de largo $\left(100 \mathrm{~m}^{2}\right)$, con una pendiente natural de 3,5\% (Figura 1). Cada una posee un embudo con un tubo colector de mampostería de $110 \mathrm{~mm}$ de diámetro que desemboca en dos piletas receptoras de escurrimientos de mampostería de 450 y 1000 litros. La segunda pileta capta $1 / 7$ del escurrimiento excedente de la primera.

El diseño del ensayo es completamente aleatorizado con 3 repeticiones por tratamiento (secuencias), que se encuentran establecidos desde el año 2006. Los tratamientos analizados fueron: soja continua (S), soja continua con trigo como cultivo de cobertura otoño-invernal (CC-S) y trigo/ soja (T/S) (Tabla 1). 


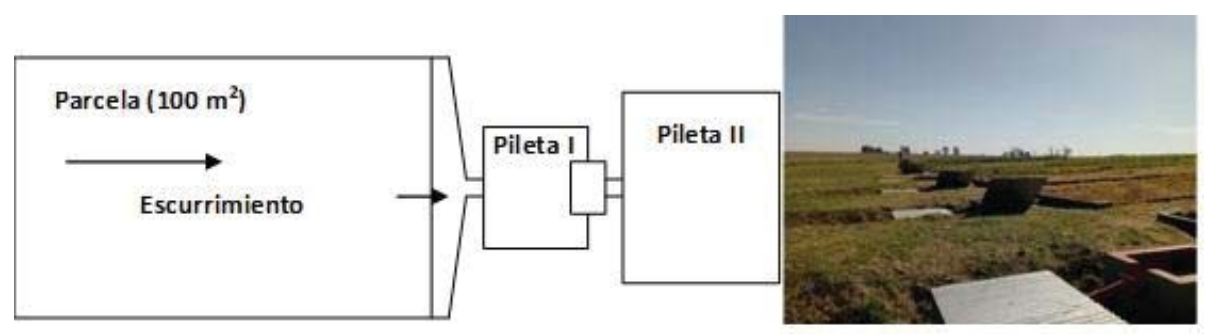

Figura 1: Foto y esquema (vista superior sin escalar) de una parcela de escurrimiento. INTA EEA Paraná.

Figure 1: Photo and diagram (unscaled top view) of a runoff plot. INTA EEA Paraná.

Tabla 1: Características principales de las secuencias de cultivo de este estudio. Table 1: Main characteristics of the crop sequences of this study.

\begin{tabular}{|c|c|}
\hline Secuencia & Características \\
\hline Soja continua & $\begin{array}{l}\text { Un cultivo productivo por campaña agrícola. Rastrojos con baja relación C/N. } \\
\text { Suelo sin cobertura vegetal viva durante la mayor parte del año. Producción } \\
\text { anual de residuos de cosecha: } 3,3 \mathrm{tn} \mathrm{ha}^{-1} \mathrm{año}^{-1}\end{array}$ \\
\hline $\begin{array}{l}\text { Soja con cultivo } \\
\text { de cobertura } \\
\text { invernal }\end{array}$ & $\begin{array}{l}\text { Un cultivo productivo por campaña agrícola. Se utiliza un cultivo de cobertura } \\
\text { para cubrir el suelo con material vivo durante el otoño-invierno y luego se lo } \\
\text { deseca para volver a implantar la soja en la siguiente campaña agrícola. } \\
\text { Producción anual de residuos de cosecha: } 6,1 \mathrm{tn} \mathrm{ha}^{-1} \text { año }\end{array}$ \\
\hline $\begin{array}{l}\text { Trigo/Soja de } \\
\text { segunda }\end{array}$ & $\begin{array}{l}\text { Dos cultivos productivos por campaña agrícola. El trigo como cultivo aporta } \\
\text { rastrojos de alta relación } \mathrm{C} / \mathrm{N} \text { a la secuencia, y mayor tiempo de cobertura al } \\
\text { suelo. Producción anual de residuos de cosecha: } 6,0 \text { tn } \mathrm{ha}^{-1} \text { año }^{-1}\end{array}$ \\
\hline
\end{tabular}

Adaptado de Oszust et al. (2018) 


\section{Determinaciones a campo $y$ en laboratorio}

El día 3/1/2019 se realizó la siembra del cultivo de soja y 7 días después, 10/1/2019, se realizó en todos los tratamientos una pulverización de glifosato $\left(2 \mathrm{~L} \mathrm{ha}^{-1}\right)$ y cleto$\operatorname{dim}\left(1 \mathrm{~L} \mathrm{ha}^{-1}\right)$. La formulación de glifosato utilizada fue concentrado soluble (54\% e.a) equivalente a $1080 \mathrm{~g} \mathrm{ha}^{-1}$; y de cletodim concentrado emulsionable ( $24 \%$ e.c) equivalente a $240 \mathrm{~g} \mathrm{ha}^{-1}$. Dos horas posteriores se registró una precipitación de $72,2 \mathrm{~mm}$, con una intensidad máxima de 153,2 $\mathrm{mm} \mathrm{h}^{-1}$.

Luego de este evento que generó escurrimiento, se procedió a determinar el volumen escurrido en el dispositivo experimental y extraer una muestra de cada parcela de escorrentía a la cual se le filtraron sedimentos. Del agua filtrada, se extrajo una alícuota que se almacenó a $-20^{\circ} \mathrm{C}$ y en condiciones de oscuridad para su posterior análisis químico. Estos fueron realizados en el laboratorio perteneciente al Programa de Investigación y Análisis de Residuos y Contaminantes Químicos (PRINARC, Santa Fe, Argentina).

Las determinaciones de glifosato $y$ AMPA se realizaron siguiendo la metodología descripta por Demonte et al. (2018), con un límite de cuantificación (LC) para ambos analitos de $0,60 \mu \mathrm{g} \mathrm{L}^{-1}$. El cletodim se analizó según el procedimiento descripto por Regaldo et al. (2017), empleando cromatografía líquida acoplada a espectrometría de masa en tándem (UHPLCMS/MS), con un $\mathrm{LC}$ de $1 \mu \mathrm{g} \mathrm{\textrm {L } ^ { - 1 }}$. La metodología completa, en todos los casos, fue validada siguiendo el documento guía de la Comunidad Europea SANTE/11945 (EC, 2015). Posteriormente se calcularon las cantidades presentes de ambos analitos en el agua de escurrimiento a partir de la multiplicación del volumen escurrido de cada parcela por las concentraciones reportadas por el laboratorio $\left(\mathrm{g} \mathrm{ha}^{-1}\right)$.
El modelo estadístico aplicado fue análisis de varianza, considerando diseño en bloques para el caso de las variables que cumplían con el criterio de normalidad y homogeneidad de la varianza (concentración y cantidades de glifosato, AMPA y cletodim en el agua de escurrimiento); mientras que para aquellas que no lo cumplían (escurrimiento y sedimentos) se siguió un análisis no paramétrico (Friedman) (InfoStat 2017). Se calcularon también los porcentajes de pérdida para cada herbicida respecto a lo aplicado y se realizaron correlaciones entre concentración y cantidad de los analitos de interés en escurrimiento y volumen escurrido y sedimentos (InfoStat 2017).

\section{RESULTADOS Y DISCUSIÓN}

Los resultados obtenidos para cada secuencia de cultivo analizada respecto a las variables de análisis pueden verse en la Tabla 2. El volumen escurrido para este evento no presentó diferencias significativas entre secuencias. En coincidencia, Sasal et al. (2010) en un estudio realizado en el mismo sitio, concluyeron que lluvias diarias mayores a $70 \mathrm{~mm}$ no muestran diferencias en el coeficiente de escurrimiento para las secuencias de cultivos bajo análisis. Sin embargo, lluvias entre 15 y $70 \mathrm{~mm}$ presentaron coeficientes de escurrimiento de diferente magnitud entre secuencias de cultivo.

En promedio, considerando los milímetros precipitados y los escurridos para todas las secuencias analizadas, el porcentaje de escurrimiento para este estudio fue $36,5 \%$ (coeficiente de escurrimiento 0,37). Los tres tratamientos analizados tenían un mismo cultivo recientemente implantado cuando ocurrió el evento de lluvia de 72,2 En 
trabajos previos considerando una escala de tiempo mayor se han caracterizado distintas variables edáficas físicas e hidrológicas asociadas a las secuencias de cultivo bajo análisis (Sasal et al., 2010; Oszust, 2018). Dichos trabajos concluyen que el tiempo en que cada sucesión de cultivos ocupa el terreno asociado al crecimiento radical resulta más relevante que la cobertura aérea como determinante del escurrimiento superficial. En este sentido, los autores resaltan significativo el índice de ocupación por cultivos (ISI) como variable explicativa de las variaciones en el escurrimiento ( $\mathrm{ISI}=\mathrm{S}<\mathrm{CC}$ / $\mathrm{S}<\mathrm{T} / \mathrm{S}$ ). Si bien en este evento aislado no hubo diferencias en escurrimiento entre tratamientos, la variable pérdida de suelo fue mayor para el monocultivo de $\mathrm{S}$ respecto a las secuencias restantes (Tabla 2).
Respecto a las pérdidas de herbicidas aplicados asociadas al escurrimiento, no se observaron diferencias entre tratamientos en la pérdida de glifosato y cletodim, sin embargo las concentraciones de AMPA fueron mayores en $\mathrm{S}$ que en $\mathrm{T} / \mathrm{S}$ (Tabla 2). La concentración y cantidad de herbicidas perdidos fue equiparable en las diferentes secuencias y esto puede estar condicionado por la elevada variabilidad que en general presentan los análisis de plaguicidas en estudios ambientales (Caprile et al., 2019; Sasal et al., 2009). Particularmente, para este estudio presentó coeficientes de variación mayores al $50 \%$ en los tres compuestos analizados. Sumado a ello, las similitudes en cuanto a la magnitud del escurrimiento entre secuencias de cultivo para este evento de precipitación mayor a $70 \mathrm{~mm}$ diario

Tabla 2: Escurrimiento y pérdidas de suelo y herbicidas en secuencias de cultivo.

Table 2: Runoff and losses of soil and herbicides in crop sequence

\begin{tabular}{|c|c|c|c|c|c|c|c|}
\hline Tratamiento & $\begin{array}{c}\text { Escurrimiento } \\
(\mathrm{mm})\end{array}$ & $\begin{array}{c}\text { Sedimentos } \\
\left(\mathrm{tn} \mathrm{ha}^{-1}\right)^{*}\end{array}$ & $\begin{array}{c}\text { Glifosato } \\
\left(\mu \mathrm{g} \mathrm{L}^{-1}\right)\end{array}$ & $\begin{array}{c}\text { AMPA } \\
\left(\mu \mathrm{g} \mathrm{L}^{-1}\right)\end{array}$ & $\begin{array}{c}\text { \% } \\
\text { Cletodim } \\
\left(\mu \mathrm{L}^{-1}\right)\end{array}$ & $\begin{array}{c}\text { pérdida } \\
\text { glifosato+ } \\
\text { AMPA }\end{array}$ & $\begin{array}{c}\text { \% pérdida } \\
\text { cletodim } \\
\star * \star *\end{array}$ \\
\hline $\mathrm{S}$ & 34,0 & $0,5 \mathrm{c}$ & 457 & $43 \mathrm{~b}$ & 46 & 13,5 & 5,9 \\
\hline CC-S & 24,0 & $0,1 \mathrm{ab}$ & 375 & $29 \mathrm{ab}$ & 43 & 9,1 & 4,4 \\
\hline T/S & 21,1 & $0,1 \mathrm{a}$ & 265 & $21 \mathrm{a}$ & 31 & 5,3 & 2,6 \\
\hline
\end{tabular}

$*(p>0,05)$ Análisis de varianza no paramétrica- Friedman

$* *(p>0,05)$. Análisis de varianza diseño en bloques

*** glifosato + AMPA en escurrimiento (cantidad)/cantidad de glifosato aplicado

****cletodim en escurrimiento (cantidad)/cantidad de cletodim aplicado 
(Sasal et al., 2010), puede explicar que se presenten también pérdidas similares de herbicidas (Oszust, 2018). De esta manera, resulta esperable que sólo se haya encontrado diferencias en el analito AMPA. Éste podría provenir de aplicaciones previas y su presencia en el suelo; variable que sí puede estar determinada por el historial de manejo de cada tratamiento. En cambio, el glifosato y cletodim encontrados en el agua de escurrimiento para este análisis estarían asociados a la aplicación realizada horas antes de la lluvia y a su inmediata escorrentía.

Los análisis de correlación entre las cantidades perdidas de ambos herbicidas, volumen de agua escurrido y sedimentos mostraron coeficientes $\mathrm{r}$ para todos los casos mayores a 0,8 . Esto resalta la importancia de continuar trabajando en aquellas prácticas que minimizan las pérdidas de agua y de sedimentos de los lotes, de manera de minimizar las pérdidas de moléculas y principios activos asociados a la producción agrícola. Particularmente resulta relevante si se considera que de acuerdo a las características físico-químicas de los plaguicidas y sus metabolitos, estos pueden ir asociados a partículas de suelo.

Las pérdidas de herbicidas por escurrimiento respecto a lo aplicado oscilaron entre $5,3 \%$ y $13,5 \%$ para glifosato y 2,6 y $5,9 \%$ para cletodim, sin diferencias significativas entre secuencias. Caprile et al. (2019) en Pergamino tampoco encontraron diferencias en esta variable para glifosato+AMPA, al comparar una rotación soja-trigo/soja-maíz con el monocultivo de soja, ambos bajo siembra directa.

Previamente, en la misma unidad de estudio de este trabajo y analizando todos los eventos de escurrimiento durante un ciclo completo del cultivo de soja, también se detectó concentraciones de glifosato y AMPA en el agua escurrida. Sin embargo, las concentraciones registradas fueron menores a las del presente trabajo: para el caso de glifosato el valor máximo fue de $12 \mu \mathrm{g} \mathrm{L}^{-1} \mathrm{y}$ para AMPA $6 \mu \mathrm{g} \mathrm{L}^{-1}$. El porcentaje perdido en función de lo aplicado durante todo el ciclo alcanzó valores de $0,05 \%$ (Sasal et al., 2009).

Sasal et al. (2013) obtuvieron picos de concentración de glifosato y AMPA (cercanos a 1500 y $400 \mu \mathrm{g} \mathrm{L}^{-1}$ respectivamente) en lluvias ocurridas 1 a 3 días después de la aplicación del herbicida, aún con muy poco volumen escurrido (precipitaciones de 30 $\mathrm{mm}$ aproximadamente). Coincidentemente, Warnemuende (2007) también detectó las mayores concentraciones de glifosato en lluvias próximas a la aplicación, con un porcentaje máximo de $7,1 \%$ respecto a lo aplicado (1 día después de la aplicación). Screpanti et al. (2005) cuantificaron los porcentajes de pérdida de glifosato por escurrimiento respecto a lo aplicado y obtuvieron resultados inferiores a los del presente trabajo $(0,031 \%$ como valor máximo). Sin embargo, en dicho estudio también se resaltó que el valor máximo fue coincidente con una lluvia que sucedió 1 día posterior a la aplicación.

Shipitalo et al. (2011) analizaron las pérdidas de herbicidas según rotaciones y métodos de labranza, encontrando también picos de concentración de glifosato en el agua de escurrimiento en lluvias posteriores a su aplicación y correlaciones significativas $(\mathrm{p}$ $<0,0001$ ) entre concentración y días posteriores a la aplicación, las cuales fueron dis- 
minuyendo a lo largo del tiempo. En dicho estudio y para el cultivo de maíz, el valor máximo de glifosato encontrado $(286 \mu \mathrm{g}$ $\mathrm{L}^{-1}$ ) fue coincidente con un escurrimiento producido 1 día después de la aplicación, mientras que para soja fue de $887 \mu \mathrm{g} \mathrm{L}^{-1}$, también coincidente con una precipitación de pocos milímetros, pero próxima a la aplicación. Los autores sostuvieron que, más importante que el tamaño del evento (mm), es la proximidad del mismo respecto a la aplicación. Esto coincide con lo hallado por Sasal et al. (2013) y González (2018). Tanto para el cultivo de maíz como soja, el porcentaje de pérdida de glifosato respecto a lo aplicado (valor promedio de todo el periodo de estudio) fue de $0,11 \%$.

En el trabajo de Shipitalo et al. (2011) detectaron que las concentraciones de AMPA en el agua de escorrentía son proporcionalmente más bajas cuando se observaron altas concentraciones de glifosato poco después de la aplicación. Esto se atribuye a un retraso en su formación a partir de su compuesto original, y estos autores mencionaron la alta correlación entre ambas concentraciones, con coeficientes de correlación de 0,87 y 0,83 para maíz y soja, respectivamente. En el presente estudio el valor de $r$ entre las concentraciones de glifosato y AMPA fue 0,75. Las cantidades perdidas de glifosato y AMPA expresadas en $\mathrm{g} 100 \mathrm{~m}^{2}$ también presentaron una alta correlación $(\mathrm{r}=0,91)$ (Figura 2). Esto avala la importancia de evaluar las pérdidas de este metabolito al considerar el impacto sobre la calidad del agua.
Las concentraciones de glifosato y cletodim presentes en el agua de escurrimiento presentaron una alta correlación $(\mathrm{r}=0,98)$ (Figura 3), dato relevante dado que no se encontraron antecedentes que lo evidencien y que se trata de herbicidas con diferente comportamiento y para este caso diferente dosis de aplicación.

No se encontraron estudios similares que evalúen al herbicida cletodim, razón por la cual no es posible establecer comparaciones para este principio activo. Estudios que avancen en este sentido son de suma importancia teniendo en cuenta el crecimiento registrado en su uso. Para la región noroeste de la provincia de Buenos Aires, un trabajo reciente realizado (Principiano, 2018) muestra que en el período abril 2017- abril 2018 dicho herbicida ocupó el $2^{\circ}$ lugar considerado la superficie aplicada, con un $11,07 \%$ (luego de glifosato con 26,3\%). Sin embargo, no se cuenta con datos para la provincia de Entre Ríos.

\section{CONCLUSIONES}

Los resultados obtenidos muestran por un lado el efecto positivo de incluir al trigo en la secuencia como cultivo para cosecha o como cobertura sobre las pérdidas de suelo vía escurrimiento superficial en comparación con el monocultivo de soja. También, la reducción en la pérdida de AMPA (concentración) vía escurrimiento superficial de la secuencia trigo/soja respecto al monocultivo de soja para este evento de precipitación analizado. Sin embargo, el re- 
M.S. Seehaus et al.

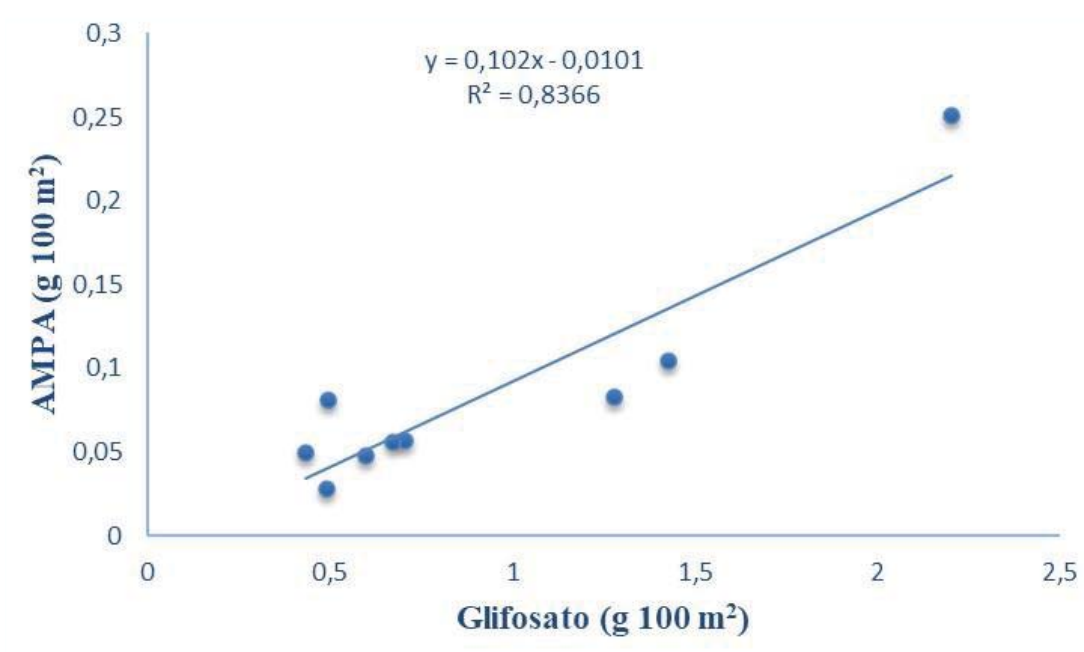

Figura 2: Correlación (Pearson) entre cantidades (g) de glifosato y AMPA encontradas en el escurrimiento.

Figure 2: Correlation (Pearson) between amounts ( $g$ ) of glyphosate and AMPA in runoff.

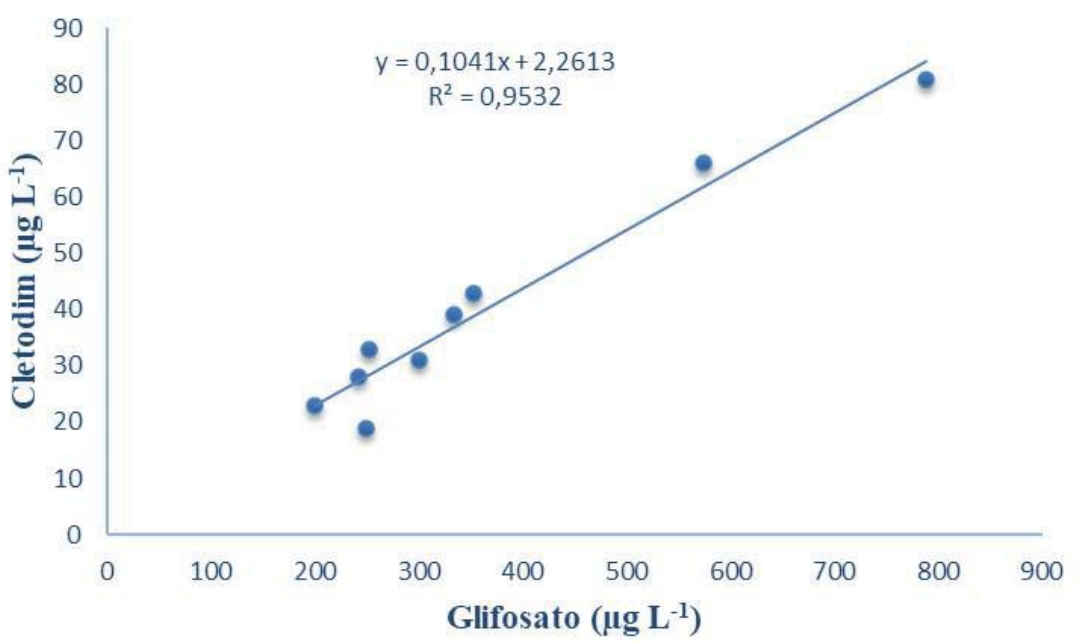

Figura 3: Correlación (Pearson) entre concentraciones $\left(\mu \mathrm{g}^{-1}\right)$ de glifosato y cletodim encontradas en el escurrimiento.

Figure 3: Correlation (Pearson) between concentration $\left(\mu \mathrm{g}^{-1}\right)$ of glyphosate and cletodim in runoff. 
sultado más destacable es la importancia de considerar las condiciones meteorológicas no sólo durante la aplicación sino en horas posteriores para reducir el riesgo de aportes difusos de agroquímicos desde lotes agrícolas hacia cuerpos de agua.

Dada la alta correlación entre las pérdidas de ambos herbicidas y suelo y agua por escurrimiento, se resalta la importancia de minimizar el escurrimiento superficial de los sistemas agrícolas con el objetivo de disminuir la cantidad de sedimentos y agua con moléculas de los insumos utilizados en la producción agropecuaria fuera del lote y hacia los cuerpos de agua. Para ello, es necesario considerar como variable esencial a las condiciones climáticas, durante la aplicación y en horas posteriores. También, considerar prácticas de conservación adicionales que reduzcan el escurrimiento y la pérdida de suelo como terrazas o que protejan cursos de agua como franjas buffer. Este enfoque requiere considerar de manera integral al sistema, y establece la necesidad de generar cambios en la toma de decisiones sobre las aplicaciones de plaguicidas en la producción agropecuaria.

\section{AGRADECIMIENTOS}

Al equipo técnico y auxiliares del departamento de Recursos Naturales y Gestión Ambiental de la Estación Experimental del INTA Paraná.

\section{BIBLIOGRAFIA}

Alonso L, Demetrio P, Etchegoyen M, Marino D. 2018. Glyphosate and atrazine in rainfall and soils in agroproductive areas of the pampas region in Argentina. Science of the Total Environment, 645: 89-96.

Andrade F, Taboada M, Lema D, Maceira N, Echeverría H, Posse G, Prieto D, Sánchez E, Ducasse D, Bogliani M, Gamundi J, Trumper E, Frana J, Perotti E, Fava F, Mastrángelo M. 2017. Los desafíos de la agricultura argentina: satisfacer las futuras demandas y reducir el impacto ambiental. Ediciones INTA, Ciudad Autónoma de Buenos Aires.

Aparicio V, De Gerónimo E, Marino D, Primost J, Carriquiriborde P, Costa JL. 2013. Environmental fate of glyphosate and aminomethylphosphonic acidin surface waters and soil of agricultural basins. Chemosphere. 93(9):1866-1873

Astoviza M. 2015. Evaluación de la distribución de contaminantes orgánicos persistentes (COPs) en aire en la zona de la cuenca del Plata mediante muestreadores pasivos artificiales. Tesis doctoral, Universidad Nacional de La Plata.

Ayarragaray M. 2015. Estudio de niveles ambientales de glifosato, ácido aminometilfosfónico (AMPA), atrazina y 2-4D en ambientes acuáticos cercanos a la ciudad de San Justo (Provincia de Santa Fe). Trabajo final de Magister en Gestión Ambiental. Facultad de Ingeniería y Ciencias Hídricas. Universidad Nacional del Litoral 104p

Battaglin WA, Meyer MT, Kuivila KM, Dietze JE, 2014. Glyphosate and its degradation product AMPA occur frequently and widely in U.S. soils, surface water, ground-water, and precipitation. J. Am. Water Resour. Assoc., 50: 275-290. 
Caprile AC, Aparicio V, Sasal C, Andriulo E. Lupi L, Bedmar F, Puricelli M, Marino D, Apa2017. Variation in glyphosate and AMPA concentrations of surface water and groundwater. In EGU General Assembly Conference Abstracts. 19: 2068.

CASAFE. 2014. Informe: Estudio de Mercado 2014 de Productos de Protección de Cultivos. Ciudad Autónoma de Buenos Aires. https:// www.casafe.org/pdf/2018/ESTADISTICAS/ Informe-Mercado-Fitosanitarios-2014.pdf

Demonte LD, Michlig N, Gaggiotti M, Adam CG, Beldoménico HR, Repetti MR. 2018. Determination of glyphosate, AMPA and glufosinate in dairy farm water from Argentina using a simplified UHPLC-MS/MS method. Sci Total Environ, 645: 34-43.

Etchegoyen A. 2014. Distribución de plaguicidas en aguas y sedimentos de fondo en los principales afluentes de la cuenca del Paraguay-Paraná. Trabajo final de Licenciatura. Facultad de Ciencias Exactas. Universidad Nacional de La Plata. p. 84.

European Commission (EC). 2015. Directorate-general for Health and Food Safety, Safety of the Food Chain Pesticides and Biocides, SANTE/11945/2015, guidance document on analytical quality control and method validation procedures for pesticides residues analysis in food and feed. Supersedes SANCO/12571/2013. Implemented by 01/01/2016.

Gonzalez JM. 2018. Runoff and losses of nutrients and herbicides under long-term conservation practices (no-till and crop rotation) in the US Midwest: A variable intensity simulated rainfall approach. International Soil and Water Conservation Research, 6(4), 265-274.

Lorenzatti EA, Negro CL, De la Sierra P, Marino F, Lenardón A. 2008. Plaguicidas en aire. Estudio preliminar en la ciudad de Santa Fe. Revista FABICIB, 12: 129-135. ricio VC, Wunderlin D, Miglioranza KS. 2019. Glyphosate runoff and its occurrence in rainwater and subsurface soil in the nearby area of agricultural fields in Argentina. Chemosphere. 225: 906-914.

Oszust JD. 2018. Calidad de agrosistemas bajo siembra directa a partir de la pérdida de nutrientes y glifosato por escorrentía. Doctorado en Ingeniería, Mención en Ciencias Agropecuarias de las Facultades de Ciencias de la Alimentación, Ciencias Agropecuarias e Ingeniería. Universidad Nacional de Entre Ríos.

Oszust JD, Ramirez AC, Sione SM, Wilson MG, Gabioud EA, Repetti MR, Sasal MC, Polla W. 2015. Evaluación de la concentración de glifosato en un embalse asociado a un ambiente agrícola en Entre Ríos. Presentado en: XXV Congreso Nacional del Agua. Paraná, Entre Ríos.

Primost J. 2013. Estudio de niveles ambientales de glifosato y AMPA en una zona modelo de intensa actividad agrícola en los alrededores de Urdinarrain, Entre Ríos. Trabajo final de Lic. en Química y Tecnología Ambiental. Facultad de Ciencias Exactas, Universidad Nacional de La Plata.

Primost JE, Marino DJ, Aparicio VC, Costa JL, Carriquiriborde P. 2017. Glyphosate and AMPA, "pseudo-persistent" pollutants under real-world agricultural management practices in the Mesopotamic Pampas agroecosystem, Argentina. Environmental Pollution, 229, 771-779.

Principiano MA, Acciaresi HA. 2018. Estado de situación del uso de herbicidas en cultivos extensivos agrícolas de la región noroeste de la provincia de Buenos Aires y el impacto 
ambiental asociado. Ediciones INTA.

Regaldo L, Gutierrez MF, Reno U, Fernández V, Gervasio S, Repetti MR, Gagneten AM. 2017. Water and sediment quality assessment in the Colastiné-Corralito stream system (Santa Fe, Argentina): impact of industry and agriculture on aquatic ecosystems. Environ Sci Pollut R. 25(7): 6951-6968.

Sasal MC, Castiglioni MG, Wilson MG. 2010. Effect of crop sequences on soil properties and runoff on natural-rainfall erosion plots under no tillage. Soil and Tillage Research. 108(1-2): 24-29.

Sasal MC, Andriulo AE, Wilson MG, Portela SI. 2010. Pérdidas de Glifosato por Drenaje y Escurrimiento en Molisoles bajo Siembra Directa. Información tecnológica, 21(5): 135-142.

Sasal MC, Wilson MG, Oszust JD, Castiglioni MG, Aandriulo AE. 2012a. Destino de fugas de N, P y glifosato del sistema agrícola. Argentina y Ambiente 2012. Mar del Plata, Argentina.

Sasal MC, Wilson, MG, Andriulo AE, Oszust JD, Gabioud EA. 2013. Estudios del impacto de la agricultura sobre la calidad del agua. In Simposio Fertilidad 2013: Intensificación Productiva Sustentable. Rosario, Argentina.

Sasal MC, Demonte L, Cislaghi A, Gabioud EA, Oszust JD, Wilson MG, Michilig N, Beldomenico HR, Repetti MR. 2015. Glyphosate loss by runoff and its relationship with phosphorous fertilization. J. Agric. Food Chem. 63 (18): 4444-4448

Sasal MC, Wilson MG, Sione SM, Beghetto SM, Gabioud EA, Oszust JD, Paravani EV, Demonte L, Repetti MR, Bedendo DJ, Medero SL, Goette JJ, Pautasso N, Schulz GA. 2017. Monitoreo de glifosato en agua superficial en Entre Ríos. La investigación acción participativa como metodología de abordaje. Revista RIA. 43 (2): 195-205.
Scotta E, Paparotti O. 1990. Aguas excedentes y tecnologías de control y captación. INTA EEA Rafaela. Publicación Misceláneas 57. Jornadas Regionales Labranzas y conservación de suelos. pp. 57-69

Screpanti C, Accinelli C, Vicari A, Catizone P. Glyphosate and glufosinate ammonium runoff from a corn-growing area in Italy. Agronomy for Sustainable Development, Springer Verlag/ EDP Sciences/INRA. 2005. 25 (3): 407-412.

Seehaus, M. 2019. Análisis socioambiental del uso de plaguicidas agrícolas en el municipio de Oro Verde (Entre Ríos, Argentina). Percepción de la población y cuantificación de la depositación atmosférica de plaguicidas. Maestría en Desarrollo Rural, Escuela para Graduados Ing. Agr. Alberto Soriano, Facultad de Agronomía, Universidad de Buenos Aires.

Shipitalo MJ, Owens LB. 2011. Comparative losses of glyphosate and selected residual herbicides in surface runoff from conservation-tilled watersheds planted with corn or soybean. Journal of environmental quality. 40(4): 1281-1289.

Shipitalo MJ, Edwards WM, Owens LB. 1997. Herbicide losses in runoff from conservation-tilled watersheds in a corn-soybean rotation. Soil Science Society of America Journal. 61(1): 267-272.

Soil Survey Staff. 1999. Soil taxonomy: A basic system of soil classification for making and interpreting soil surveys. 2nd edition. Natural Resources Conservation Service. U.S. Department of Agriculture Handbook.

Taberner Palou A, CirujedaRanzenberger A, Zaragoza Larios C. 2007. Manejo de poblaciones de malezas resistentes a herbicidas. 100 preguntas sobre resistencias. Organización de las Naciones Unidas para la Agricultura y la Alimentación, Roma. [Consultado: 18/09/2018]. http://www.fao.org/3/a-a1422s. pdf 


\section{M.S. Seehaus et al.}

Ustarroz D, Montoya J, Ledda A, Belluccini PA, Cervellini JM. 2017. Actividad de cletodim y haloxifop R-metil en el suelo y su efecto fitotóxico en el cultivo de maíz (Zea mays). AgriScientia. 34(2): 83-89.

Vazquez-Amabile G, Ricca AP, Rojas D, Cristos D, Ortiz ML, de Zarate GP, ... Rodriguez-Vagaria A. 2017. Análisis de agroquímicos y nitratos en cursos y aguas subterránea de cuencas rurales del oeste y sudeste de Buenos Aires. Productividad y medio ambiente. 61.

Villaamil Lepori EC, Bovi Mitre G, Nassetta M. 2013. Situación actual de la contaminación por plaguicidas en Argentina. Revista Internacional de Contaminación Ambiental, 29: 25-43

Villarreal R, Vittori S, Laoretani EA, Lozano LA, Soracco CG. 2018. Dinámica del glifosato y ampa en suelos bajo siembra directa y labranza convencional. Investigación Joven, 4.

Warnemuende EA, Patterson JP, Smith DR, Huang CH. 2007. Effects of tilling no-till soil on losses of atrazine and glyphosate to runoff water under variable intensity simulated rainfall. Soil and Tillage Research. 95(12): $19-26$ 